\title{
Commissioning of the ACtive TARget and Time Projection Chamber (ACTAR TPC)
}

\author{
B. Mauss ${ }^{\mathrm{a}, *, 1}$, P. Morfouace ${ }^{\mathrm{a}}$, T. Roger ${ }^{\mathrm{a}, *}$, J. Pancin $^{\mathrm{a}}$, G.F. Grinyer ${ }^{\mathrm{a}, \mathrm{b}}$, J. Giovinazzo $^{\mathrm{c}}$, \\ V. Alcindor ${ }^{a}$, H. Álvarez-Pol ${ }^{d}$, A. Arokiaraj ${ }^{\mathrm{e}}$, M. Babo ${ }^{\mathrm{f}}$, B. Bastin $^{\mathrm{a}}$, C. Borcea $^{\mathrm{g}}$, M. Caamaño $^{\mathrm{d}}$, \\ S. Ceruti ${ }^{\mathrm{e}}$, B. Fernández-Domínguez ${ }^{\mathrm{d}}$, E. Foulon-Moret $^{\mathrm{a}}$, P. Gangnant ${ }^{\mathrm{a}}$, , S. Giraud $^{\mathrm{a}}$, , A. Laffoley $^{\mathrm{h}}$, \\ G. Mantovani ${ }^{i}$, T. Marchi ${ }^{i}$, B. Monteagudo ${ }^{j}$, J. Pibernat $^{c}$, O. Poleshchuk ${ }^{\mathrm{e}}$, R. Raabe ${ }^{\mathrm{e}}$, \\ J. Refsgaard ${ }^{\mathrm{e}}$, A. Revel ${ }^{\mathrm{a}, \mathrm{j}}$, F. Saillant ${ }^{\mathrm{a}}$, M. Stanoiu ${ }^{\mathrm{g}}$, G. Wittwer ${ }^{\mathrm{a}}$, J. Yang ${ }^{\mathrm{e}}$ \\ a Grand Accélérateur National d'Ions Lourds (GANIL), CEA/DRF-CNRS/IN2P3, Bvd Henri Becquerel, 14076 Caen, France \\ ${ }^{\mathrm{b}}$ Department of Physics, University of Regina, Regina, SK S4S OA2, Canada \\ ' Centre d'Études Nucléaires de Bordeaux Gradignan (CENBG) - UMR 5797, CNRS/IN2P3 - Université de Bordeaux, Chemin du \\ Solarium, F-33175 Gradignan, France \\ ${ }^{\mathrm{d}}$ Dpt. de Física de Partículas Univ. of Santiago de Compostela and IGFAE, E-15758, Santiago de Compostela, Spain \\ ${ }^{\mathrm{e}} \mathrm{KU}$ Leuven, Instituut voor Kern- en Stralingsfysica, 3001 Leuven, Belgium \\ ${ }_{\mathrm{f}}^{\mathrm{f}}$ Institut de Physique Nucléaire, CNRS/IN2P3, Université Paris-Sud, Université Paris-Saclay, F-91406 Orsay, France \\ ${ }^{g}$ IFIN - HH, str. Reactorului 30, P. O. Box MG - 6, Bucharest-Magurele, Romania \\ ${ }^{\mathrm{h}}$ Department of Physics, University of Guelph, Guelph, ON N1G 2W1, Canada \\ ${ }^{i}$ INFN — Laboratori Nazionali di Legnaro, 35020 Legnaro, Italy \\ ${ }^{j}$ LPC Caen, Normandie Université, ENSICAEN, Université de Caen, CNRS/IN2P3, F-14050, Caen, France
}

\section{A R T I C L E I N F O}

\section{Keywords:}

ACtive TARget

Time Projection Chamber

MICROMEGAS

Resonant scattering

\begin{abstract}
A B S T R A C T
The ACtive TARget and Time Projection Chamber (ACTAR TPC) is a novel gas-filled detector that has recently been constructed at GANIL. This versatile detector is a gaseous thick target that allows the tracking of charged particles in three dimensions and provides a precise reaction energy reconstruction from the vertex position. A commissioning experiment using resonant scattering of a $3.2 \mathrm{MeV} /$ nucleon $^{18} \mathrm{O}$ beam on an isobutane gas (proton) target was performed. The beam and the heavy scattered ions were stopped in the gas volume, while the light recoil left the active volume and were stopped in auxiliary silicon detectors. A dedicated tracking algorithm was applied to determine the angle of emission and the length of the trajectory of the ions, to reconstruct the reaction kinematics used to built the excitation functions of the ${ }^{1} \mathrm{H}\left({ }^{18} \mathrm{O},{ }^{18} \mathrm{O}\right){ }^{1} \mathrm{H}$ and ${ }^{1} \mathrm{H}\left({ }^{18} \mathrm{O},{ }^{15} \mathrm{~N}\right){ }^{4} \mathrm{He}$ reactions. In this article, we describe the design of the detector and the data analysis, that resulted in center of mass reaction energy resolutions of 38(4) keV FWHM and 54(9) keV FWHM for the proton and alpha channels, respectively.
\end{abstract}

\section{Introduction}

The study of nuclear matter far from the valley of stability has led to significant changes in terms of techniques used to study exotic nuclei when compared to studies of stable nuclei. The dramatic decrease in intensity for radioactive ion beams, and the inherent need to work in inverse kinematics (the beam is the heavy particle, as no target can be made with short lived radioactive nuclei), has to be compensated with the use of thicker targets. This, unfortunately, decreases the overall resolution that can be obtained on the determination of the final state parameters, such as the excitation energy. To cope with these challenges, active targets were developed a few decades ago as summarized in a recent review article [1]. These detectors are usually based on the concept of Time Projection Chambers (TPC). Using the detection medium as a target for nuclear reactions, they are used to track charged particles, extract reaction vertices and thus correct the energy lost by charged particles inside the target. The first active target built for nuclear physics experiments was the IKAR setup at GSI [2], which was constructed to study exotic light-ion matter distributions. Since then, more versatile instruments were developed such as the active target MAYA at GANIL [3] that was used to study transfer reactions on light exotic nuclei [4-6] and giant resonances in Ni isotopes [7,8]. At about the same time, the CENBG TPC [9] was developed to investigate exotic

\footnotetext{
* Corresponding authors.

E-mail addresses: benoit.mauss@riken.jp (B. Mauss), roger@ganil.fr (T. Roger).

1 Present address: RIKEN Nishina Center, 2-1, Hirosawa, Wako, Saitama 351-0198, Japan.
} 
radioactivity modes, such as two proton radioactivity [10]. With the advent of new digital electronics developments, such as the General Electronics for TPCs project (GET) [11], next generation active targets and time projection chambers with more precise volume sampling have been constructed or are planned worldwide [1,12-15].

The ACtive TARget and Time Projection Chamber (ACTAR TPC) is a state-of-the-art detector developed by a collaboration of European laboratories and built at GANIL. It has a charge projection plane segmented in $128 \times 128$ square pads of $2 \times 2 \mathrm{~mm}^{2}$ connected to the GET system. With GET, the signal can be digitized at several user-specified frequencies in 512 time cells per channel, sampling the active volume in 8 Mega voxels. This article presents the performances of ACTAR TPC using the ${ }^{1} \mathrm{H}\left({ }^{18} \mathrm{O},{ }^{18} \mathrm{O}\right){ }^{1} \mathrm{H}$ and ${ }^{1} \mathrm{H}\left({ }^{18} \mathrm{O},{ }^{15} \mathrm{~N}\right){ }^{4} \mathrm{He}$ resonant reactions performed during a 20 -hour commissioning experiment at GANIL.

\section{Experimental set-up}

\subsection{Detector description}

The ACTAR TPC detector, shown schematically in Fig. 1, follows the construction of two 8-times smaller demonstrator [16,17] using different technologies for the pad wiring. The internal skeleton of ACTAR TPC chamber measures $606 \mathrm{~mm} \times 606 \mathrm{~mm} \times 335 \mathrm{~mm}$. The gas input and output are positioned on opposite corners of the chamber to ensure a good overall homogeneity of the gas or gas mixtures. All flanges around the structure are made with $1.5 \mathrm{~cm}$ thick aluminum in order to sustain up to one bar differential pressure with minimum deformation. In order to put the $6 \mu \mathrm{m}$ thick Mylar beam-entrance window as close as possible to the wire field cage, the front flange is equipped with a cylindrical nose with a diameter of $80 \mathrm{~mm}$. This design reduces the distance from the entrance window to the active volume of the detector to only $60 \mathrm{~mm}$, as shown in Fig. 1. The bottom and top flanges are used to mechanically support the drift cage and provide the necessary connections to the pixelated anode, respectively. The side flanges can be used to hold auxiliary detectors, depending on the needs of the particular physics experiments.

The active volume is surrounded by a $295 \mathrm{~mm} \times 295 \mathrm{~mm} \times 255 \mathrm{~mm}$ double-wire field cage that is attached to the cathode and is fed via a $20 \mathrm{kV}$ high voltage feedthrough on the bottom flange. The field cage consists of $20 \mu \mathrm{m}$ diameter wires with a $1 \mathrm{~mm}$ pitch on the inner plane and a $2 \mathrm{~mm}$ pitch on the outer plane, connected through $4.7 \mathrm{M} \Omega$ resistors. As explained in Ref. [16], the use of two field degradation planes ensures a good homogeneity of the drift field by preventing the electric field between the drift cage and the auxiliary detectors to leak into the drift region.

With the drift electric field, ionization electrons produced in the active region drift towards the anode that is highly segmented into $128 \times 128$ square pads, each with a side edge of $2 \mathrm{~mm}$. The resulting high density of channels (25 channels $/ \mathrm{cm}^{2}, 16384$ total pads) was therefore a challenge for designing the mechanics and pad connections while ensuring minimal mechanical deformation of the flange when applying up to 1 bar differential pressure. Typical experiments are performed with pressures ranging from a few tens of mbar up to one bar. Our solution was to build the anode from a metal-core printed circuit board (PCB) with a direct connection through the circuit from the pads to a connector with a $2 \mathrm{~mm}$ pitch (size of a pad), as described in Ref. [17].

Based on the demonstrator detector design [16], the pad plane for ACTAR TPC was equipped with $\mathrm{a} \simeq 220 \mu \mathrm{m}$ gap bulked MICROMEGAS amplification system $[18,19]$ made by the CERN PCB Workshop. Usually, the amplification gap in MICROMEGAS is about $100 \mu \mathrm{m}$, which is the optimum gap for atmospheric pressure operation. Here, the large amplification gap was chosen based on the study in Ref. [20] where good amplification was achieved even at low pressures (below 300 mbar).
The 16384 pads are connected through flex Kapton spark-protection circuits (ZAP) to the 64 ASIC and ADC (AsAd) boards of the GET system. The AsAd are arranged perpendicular to the beam axis in order to minimize the amount of channels per AsAd board hit by the beam, hence reducing the dead time as explained in Ref. [11]. The AsAd boards are connected to $8+8$ Concentration Boards $(\mathrm{CoBo})$ cards held in two Micro Telecommunications Computing Architecture ( $\mu$ TCA) crates. Two Multiplicity, Trigger and Time (MuTanT) modules (one master and one slave) are used to distribute the $100 \mathrm{MHz}$ clock to the CoBo boards, and distribute the trigger to GET. The $\mu$ TCA crates carrier hubs $(\mathrm{MCH})$ are connected through their $10 \mathrm{~Gb}$ interface to a PowerEdge R730xd Dell acquisition server equipped with two Intel Xeon E5-2630 v4 central processing units (CPU).

For the present study, as seen in Fig. 1, the downstream flange of ACTAR TPC was covered with twenty $5 \times 5 \mathrm{~cm}^{2}$ and $700 \mu \mathrm{m}$ thick silicon detectors $(\mathrm{Si})$ that were used to detect the scattered protons escaping the active volume. They were each equipped with $10 \mathrm{mV} / \mathrm{MeV}$ charge-sensitive preamplifiers that fed CAEN N568 amplifiers with $3 \mu$ s shaping time and $\times 32$ gain. The shaped output of the module was connected to GANIL VXI peak-sensing ADCs. The fast output of the amplifiers was discriminated in CAEN N844 modules and used to trigger the GAMER VXI trigger module. The VXI electronics coupling to the GET system was made using the CENTRUM protocol [21] with the GAMER VXI used as master trigger for the whole electronics setup.

\subsection{Settings of the commissioning}

The ACTAR TPC commissioning experiment was performed at GANIL. The goal was to estimate the capabilities of the detector for performing resonant scattering studies. A low-energy ${ }^{18} \mathrm{O}$ beam was chosen to benchmark the resolution of the detector based on the reconstruction of the excitation function of the ${ }^{1} \mathrm{H}\left({ }^{18} \mathrm{O},{ }^{18} \mathrm{O}\right){ }^{1} \mathrm{H}$ and ${ }^{1} \mathrm{H}\left({ }^{18} \mathrm{O},{ }^{15} \mathrm{~N}\right){ }^{4} \mathrm{He}$ reactions.

An ${ }^{18} \mathrm{O}$ beam was accelerated to $6.6 \mathrm{MeV} /$ nucleon in the CSS1 cyclotron and was sent to the G3 experimental hall where ACTAR TPC was mounted on the beam line. The beam was slowed down using a $63 \mu \mathrm{m}$ thick aluminum degrader and reached the beginning of the active volume with an energy of $3.2 \mathrm{MeV} /$ nucleon. This lower energy allowed states in ${ }^{19} \mathrm{~F}$ to be populated between 8 and $11 \mathrm{MeV}$ in excitation energy. The incoming intensity was monitored using a collimated multiwire proportional counter (MWPC) that was placed in front of the entrance window of ACTAR TPC. During the $20 \mathrm{~h}$ of data taking, the average ${ }^{18} \mathrm{O}$ beam intensity was $1.5 \times 10^{4}$ particles per second. The proton target used to study the ${ }^{1} \mathrm{H}\left({ }^{18} \mathrm{O},{ }^{18} \mathrm{O}\right){ }^{1} \mathrm{H}$ and ${ }^{1} \mathrm{H}\left({ }^{18} \mathrm{O},{ }^{15} \mathrm{~N}\right){ }^{4} \mathrm{He}$ reactions was isobutane $\left(\mathrm{iC}_{4} \mathrm{H}_{10}\right)$ gas at $100 \mathrm{mbar}$ pressure that filled the ACTAR TPC volume. At this pressure, the ${ }^{18} \mathrm{O}$ beam was stopped inside the active volume, just above column number 98 (out of 128 total) of the pad plane. The maximum energy of the scattered protons was about $12 \mathrm{MeV}$, at $0^{\circ}$ in the laboratory frame. The corresponding energy deposited in the gas was $\frac{d E}{d x}=1 \mathrm{keV} / \mathrm{mm}(2 \mathrm{keV} / \mathrm{pad})$, which is the minimum energy deposit in the experiment. With the MICROMEGAS mesh voltage set to $-450 \mathrm{~V}$, the gain was previously measured to be 5000 in Ref. [16]. Hence, all proton tracks can be recorded with a transverse multiplicity of three pads. This is necessary to achieve an optimal angle reconstruction, and a good signal-to-noise ratio using the $120 \mathrm{fC}$ GET preamplifier dynamic range, which corresponds to a gain of $180 \mathrm{mV} / \mathrm{MeV}$ Si equivalent. However, the difference in energy deposit between ${ }^{18} \mathrm{O}$ ions stopping in the active volume (about $100 \mathrm{keV} / \mathrm{mm}$ ) and the recoiling protons was up to a factor 100 .

As shown in Ref. [16], a single dynamic range of the GET preamplifiers is not sufficient to record the track of the beam ions and the track of the protons at the same time without saturating the electronics. In order to extend the effective dynamic range, the pads located below the beam path were polarized to $-100 \mathrm{~V}$, locally reducing the gain of the MICROMEGAS from about 5000 to about 250. This follows the work described in Ref. [22]. 


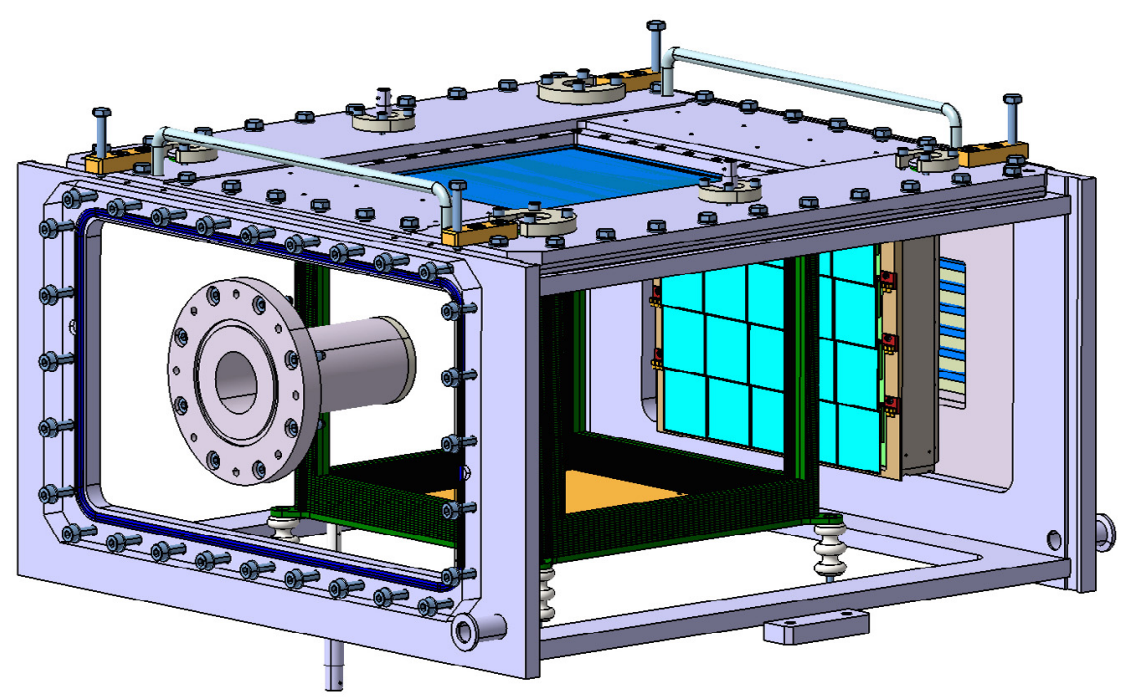

Fig. 1. 3D computer-aided drafting (CAD) drawing of ACTAR TPC. See text for details.
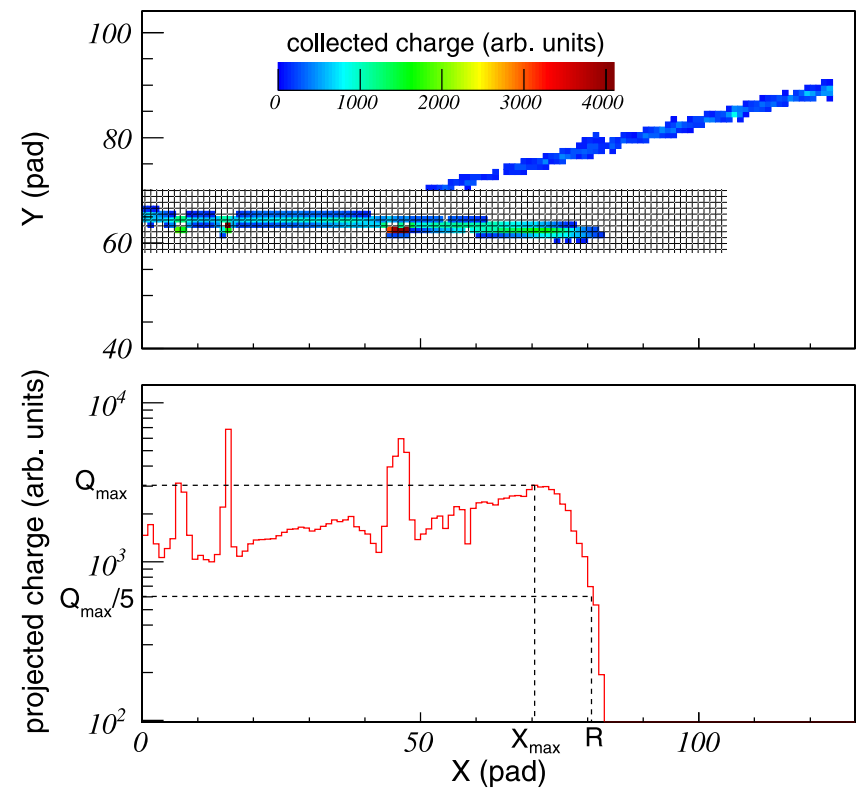

Fig. 2. (Color online) Upper panel, example of an event with the shaded polarization zone below the beam. Lower panel, projection of the beam particle along the $\mathrm{X}$ axis. The track stopping point $R$ of the beam, plus the scattered heavy particle if there is a reaction as it is the case here, is defined as a fifth of the Bragg curve maximum charge deposit. The polarization could not be applied for some pads, due to connection problems, resulting in a significantly higher charge compared to their neighbors (around $X=10$ pad and $X=50$ pad), which results in the peaks shown in the reconstructed Bragg curve. These pads were discarded in the analysis.

With a beam emittance of about $\pi \sigma_{x} \sigma_{\theta}=18.56 \pi \mathrm{mm} \cdot \mathrm{mrad}$, obtained by tracking the beam upstream from the reaction point, the width of the polarized zone was set to 12 rows of pads $(24 \mathrm{~mm})$ and was 100 columns $(200 \mathrm{~mm})$ in length, as shown in the upper panel of Fig. 2. Due to connection problems, some of the pads from this zone could not be correctly polarized. As a result, the MICROMEGAs has a higher gain above those pads, which produces the peaks on the Bragg profile shown in the lower panel of Fig. 2. The GET system was set to partial readout mode, so that only pads with a signal exceeding a certain threshold were read. This readout threshold was set to $5.3 \mathrm{fC}$, which corresponds to $3.27 \times 10^{4}$ electrons. With the MicRomegas gain of 5000, this threshold is about 6.6 ionization electrons, or about $0.2 \mathrm{keV}$ energy deposited per pad.
Table 1

Beam and detector settings used during the $\mathrm{ACTAR} \mathrm{TPC}^{18} \mathrm{O}$ commissioning experiment.

\begin{tabular}{lll}
\hline Parameter & Value & Units \\
\hline Beam energy at active volume entrance & 57.6 & $\mathrm{MeV}$ \\
Beam emittance & $18.56 \pi$ & $\mathrm{mm} \cdot \mathrm{mrad}$ \\
Average beam intensity & $1.5 \times 10^{4}$ & $\mathrm{pps}$ \\
Cathode potential & -3500 & $\mathrm{~V}$ \\
Mesh potential & -450 & $\mathrm{~V}$ \\
Polarized pads potential & -100 & $\mathrm{~V}$ \\
Drift velocity (measured) & 3.8 & $\mathrm{~cm} / \mu \mathrm{s}$ \\
Isobutane gas pressure & 100 & $\mathrm{mbar}$ \\
Proton target density & $2.5 \times 10^{19}$ & $\mathrm{protons} / \mathrm{cm}^{3}$ \\
Clock frequency & 50 & $\mathrm{MHz}$ \\
GET dynamic range & 120 & $\mathrm{fC}$ \\
GET readout threshold & 5.3 & $\mathrm{fC}$ \\
GET peaking time & 1024 & $\mathrm{~ns}$ \\
\hline
\end{tabular}

The cathode voltage was set to $-3500 \mathrm{~V}$, so that the drift velocity of the ionization electrons was about $3.6 \mathrm{~cm} / \mu \mathrm{s}$. The total time window required to observe the full height of the active volume was therefore $6.7 \mu \mathrm{s}$. The sampling frequency of the GET system was set to $50 \mathrm{MHz}$, which provided a time window of $10.24 \mu$ s over 512 samples for detecting the ionization electrons. Following the definition of Ref. [23], the samples will be later referred to as time cells. The test experimental conditions are summarized in Table 1.

For later discussions on the analysis, we introduce the following terms:

- Electronic events are defined as the full window of 512 time cells over the whole pad plane.

- Physical events are defined as the grouping of tracks correlated in time/space.

- Beam events are defined as unreacted events, forming physical events only consisting of a beam track.

- Pileup events are defined when there are several physical events in an electronic event.

\section{Data analysis}

\subsection{Particle tracking}

To extract the voxel information (the charge collected and the electron arrival time on each pad), the signal baseline was first treated using the methods described in Ref. [23]. After baseline treatment, the 


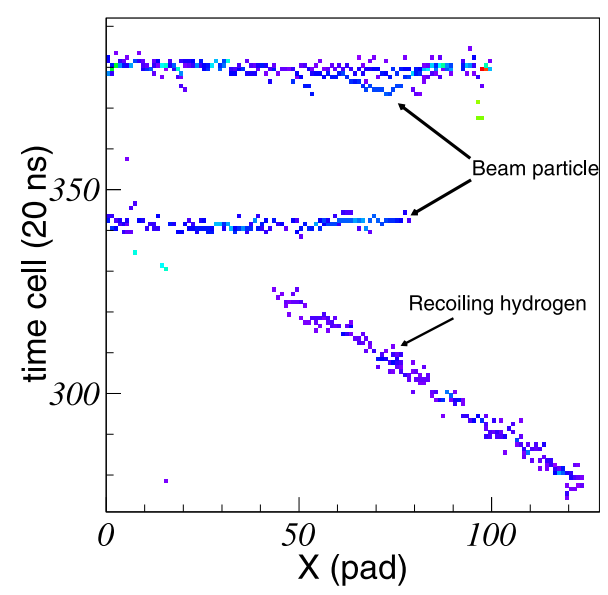

Fig. 3. (Color online) Example of pileup event observed in ACTAR TPC, two beam tracks are seen and only one recoil, corresponding to a reaction. The beam tracks that reacted has a shorter track. The beam track above corresponds to a beam event happening at a later time than the physical event below. The color scale indicates the charge deposit.

physical pulse was fit with a generic pulse shape as described in [24]. The timing information was translated into a vertical position using the electron drift velocity in the gas. By correlating the Si detector hit with the recoil nuclei tracked, the impact matrix on each Si detector can be reconstructed. The drift velocity is adjusted to match the reconstructed vertical size of the Si detector to $5 \mathrm{~cm}$. The determined value of $3.8 \mathrm{~cm} / \mu \mathrm{s}$ is in agreement with the MAGBoLTz [25] calculated velocity of $3.6 \mathrm{~cm} / \mu \mathrm{s}$.

A typical event contains the beam track and the track of the heavy scattered ion that stops in the active volume. Given that the maximum scattering angle of the heavy ion does not exceed $15^{\circ}$ for ( $\left.p, \alpha\right)$ reactions at the lowest measured reaction energy, the tracks directions are very close and are almost always confined to the polarized (low gain) region of the pad plane. For this reason, we consider in the analysis and define in the following that beam tracks are continuous tracks in the polarized beam region (Fig. 2), meaning unreacted beam or the incoming beam plus the scattered heavy-ion, without distinction. Scattering events also contain the track of the light recoil particle that is observed in the unpolarized (high gain) zone. As seen in the upper panel of Fig. 2, the pad plane can thus be separated into two areas: the polarized zone, that contains the tracks of the heavy ions, and the unpolarized zone that contains the tracks of the light recoils. Following this division, the tracking analysis is performed in each area independently. The track fitting procedure is adapted from the orthogonal distance regression presented in Ref. [26].

Due to the high beam intensity, the probability to record two incident beam particles in the same acquisition window of $10.24 \mu \mathrm{s}$ is significant. A pileup separation technique was therefore developed and applied to the polarized zone. Pileup events arrive at different times and are thus separated into different time cells, as shown in the example of Fig. 3, which displays the charge projection of three particle tracks in the $\mathrm{X}-\mathrm{Z}$ plane $(\mathrm{X}$ is the axis of propagation of the beam and $\mathrm{Z}$ the vertical axis along which the electrons drift). Comparing the recoiling particle timing (the track going down in Fig. 3) and timing of the two separate beam tracks (horizontal tracks) in a single electronic event, the recoil and one beam track can be regrouped in a correlated physical event. In Fig. 4, the amount of pileup per electronic event is plotted as a function of the beam intensity. For example, at a beam intensity of $10^{4}$ particle per second (pps, left panel of Fig. 4), only $12 \%$ of the electronic events recorded more than one beam track. When the beam intensity was doubled to $2 \times 10^{4}$ pps (right pane of Fig. 4), $27 \%$ of the events were piled up. This is consistent with the expected Poisson time distribution of the beam. Using the pileup separation technique, close to $100 \%$ of these pileup events were recovered in the analysis. The separation limit is given by the time difference between two beam tracks compared to the beam track time width, due to the longitudinal straggling of the electrons as they drift toward the MICROMEGAs. In this commissioning, this width was consistent with the MAGBOLTZ calculation, that depends on the gas used and the magnitude of the drift electric field applied.

\subsection{Particle identification}

After tracking particles in the TPC, the second step of the analysis consisted in identifying the light recoil. This identification was performed by correlating the energy recorded in the silicon detectors $E_{\mathrm{Si}}$ with the energy loss in the gas per millimeter, averaged over the last 15 columns of pads. However, the measurement of the energy deposition in the gas is affected by attachment due to $\mathrm{O}_{2}$ molecules. Indeed, during the experiment, a small air leak on the pad plane flange was detected. In order to estimate the proportion of air in the detector, several short runs were taken using the MWPC in front of ACTAR TPC as trigger, allowing the selection of unreacted beam events by looking at events without energy deposit in the Si. The range of the beam particles was compared to LISE++ calculations [27] using the energy loss tables of Ref. [28]. A $2 \%$ air pollution was deduced and was found to be constant over the 20hour experiment. Drift electron attachment to the $\mathrm{O}_{2}$ molecules caused the charge recorded over the pad plane to depend exponentially on the position (height above the anode) in which the ionization electrons were produced. The information on the energy deposited had to be corrected for this effect by applying a factor that varied exponentially with the drift distance. With the silicon trigger, the number of time cell can be directly transformed into a drift distance for the trigging event. Then, the exponential correction parameters were determined from the correlation between the energy loss measured over the pad plane of $25 \mathrm{MeV} \alpha$ particles recorded in the silicon detectors, and the known mean drift distance of their tracks. $\alpha$ particles are identified by the fact that the proton punch-through is at $10 \mathrm{MeV}$ and only negligible amount of other particles are expected to reach the silicon detectors. The correlation is shown in the inset of Fig. 5, with the exponential fit (correction factor) drawn through the data points. The attachment parameter extracted from a fit of this 2D plot reaches $\lambda_{a}=0.126 \mathrm{~cm}^{-1}$, about a factor 10 above the value deduced with MAGBOLTz. After applying this correction, the identification plot presented in Fig. 5 was obtained. Without the correction factor, the identification lines width in $\frac{d E}{d x}$ would spread about four times more.

\subsection{Reaction channel selection}

In the present experiment, four reaction channels are open. Two of them have a proton and an ${ }^{18} \mathrm{O}$ in the final state: the elastic channel and the inelastic channel to the first excited state of ${ }^{18} \mathrm{O}$ in the highest energy part of the excitation function. Two other reactions have an $\alpha$ in the final state together with a ${ }^{15} \mathrm{~N}$ either in its ground state or in its first excited state. If the particle identification can be achieved with the classic thick solid target in inverse kinematics method [29,30], active targets offer a unique possibility to select the excitation energy in the final state. This is achieved through the tracking of the heavy particle, or more precisely, by the determination of the total path of the beam plus scattered heavy particle after reaction in the active volume $R$. This can be extracted by projecting the charge collected on the pad plane polarized area along the $X$-axis (Fig. 2). The beam energyloss profile along the $X$-axis is constructed and the beam-like particle stopping point is determined. As defined in the lower panel of Fig. 2, it corresponds to the point where the charge value has decreased to a fifth of the Bragg peak maximum charge, which corresponded best to the SRIM energy loss tables [31].

As explained in [32,33], if the center-of-mass angle of the reaction is fixed, there is a unique relationship between $E_{\mathrm{Si}}$ and $R$ to the reaction 

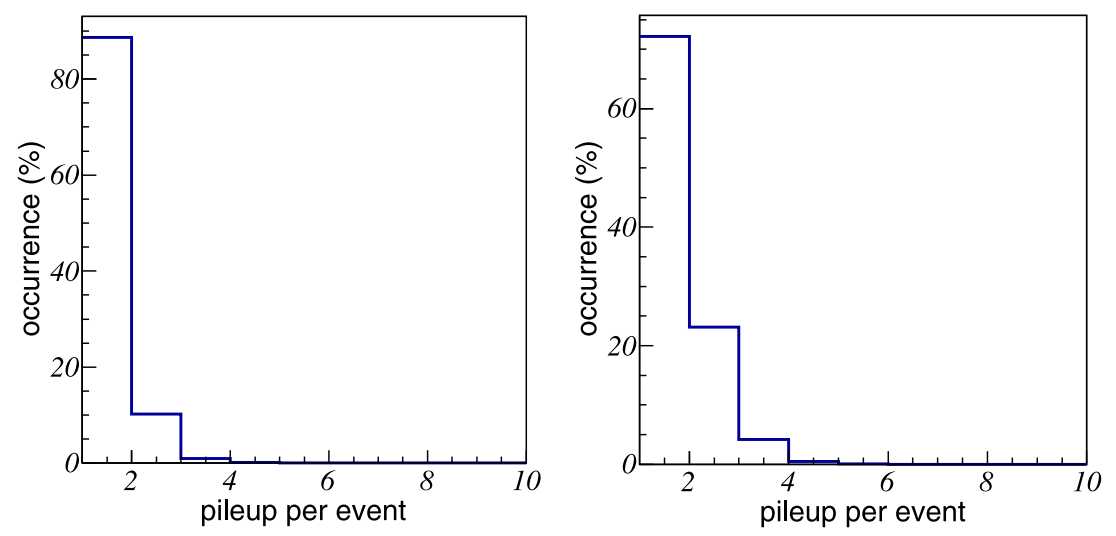

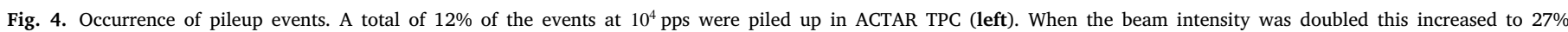
(right). Most of these events were recovered in the analysis by correlating the timing between the light recoil to one of the incident beam tracks (see text).

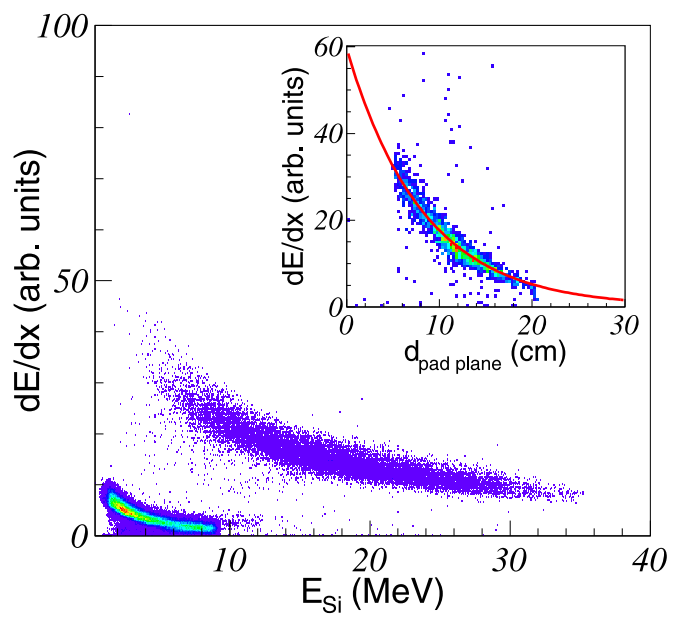

Fig. 5. (Color online) Particle identification using the energy deposit over the pad plane versus the energy lost in the silicon detectors after correction of the height dependence. The energy deposit is averaged over the last 15 columns of pads. The identification lines observed correspond to hydrogen and helium. In the inset, the correlation of the energy deposit over the pad plane with respect to the distance between the pad plane and the hit position on the $\mathrm{Si}$ detector extracted from the track fit is plotted for $E_{\mathrm{Si}}=25 \pm 1 \mathrm{MeV}$. The solid red line corresponds to the applied correction function.

energy and the excitation energy in the final state. Correlation plots for ${ }^{1} \mathrm{H}\left({ }^{18} \mathrm{O},{ }^{18} \mathrm{O}\right){ }^{1} \mathrm{H}$ and ${ }^{1} \mathrm{H}\left({ }^{18} \mathrm{O},{ }^{15} \mathrm{~N}\right){ }^{4} \mathrm{He}$ are presented in Fig. 6 for $160 \pm 5^{\circ}$ center-of-mass, selected with the proton or $\alpha$-particle laboratory angle. This angle was chosen for comparison with the measurements of Ref. [34]. The zero of the abscissa corresponds to the start of the pad plane. The events corresponding to the population of the ground state of ${ }^{18} \mathrm{O}$ and ${ }^{15} \mathrm{~N}$ can be identified on the figure. Though energetically allowed, no events corresponding to the population of the first excited state of ${ }^{18} \mathrm{O}$ and ${ }^{15} \mathrm{~N}$ were observed. This is consistent with the measurement of Ref. [34]. The vertical lines observed in Fig. 6 come from pileup events when $R \simeq 20 \mathrm{~cm}$. Below $20 \mathrm{~cm}$, they come from the information lost due to the unpolarized pads described previously and shown in Fig. 2. On the right of Fig. 6, points can also be observed below the ${ }^{15} \mathrm{~N}$ ground state line. Most of these correspond to fusion reactions with ${ }^{12} \mathrm{C}$ in the $\mathrm{iC}_{4} \mathrm{H}_{10}$ gas and evaporation of an $\alpha$-particle. They can be partially identified because the Bragg peak maximum of ${ }^{26} \mathrm{Mg}$ is about $80 \%$ larger than ${ }^{15} \mathrm{~N}$. This identification can be tentatively performed for reactions before pad 50. Beyond that point, the reaction energies are below $20 \mathrm{MeV}$. At the center-of-mass angles used in the analysis, this corresponds to ${ }^{26} \mathrm{Mg}$ with an energy below $2.5 \mathrm{MeV}$, beyond the Bragg peak. The energy deposited by ${ }^{26} \mathrm{Mg}$ is then indistinguishable from ${ }^{15} \mathrm{~N}$. In this case, identification of the heavy particle before pad
50 is not performed because of the unpolarized pads in the beam area (Fig. 2). As fusion-evaporation is not kinematically allowed above the ${ }^{1} \mathrm{H}\left({ }^{18} \mathrm{O},{ }^{15} \mathrm{~N}\right){ }^{4} \mathrm{He}$ ground state kinematic line, these reactions provide a source of background that can be largely suppressed by selecting the ${ }^{15} \mathrm{~N}$ ground-state line.

\subsection{Construction of the excitation function}

After selecting the reaction channel, the reaction energy was reconstructed using an iterative procedure. The reaction kinematics was used to calculate the reaction energies. Having the energy deposited in the Si detectors $E_{\mathrm{Si}}$ and the track angle $\theta_{\text {lab }}$ fitted from the TPC voxels, the reaction energy $E_{\text {reac }}$ was determined using the formula:

$E_{\mathrm{reac}, i}=\frac{m_{{ }_{18} \mathrm{O}}\left(m_{\mathrm{H}} / m_{18} \mathrm{O}+1\right)^{2} E_{\mathrm{H}, i}}{4 m_{\mathrm{H}} \cos ^{2}\left(\theta_{\mathrm{lab}}\right)}$

for the case of the (p,p) channel, with $i$ the iteration number. $m_{\mathrm{H}}$ and $m_{18} \mathrm{O}$ are the proton and ${ }^{18} \mathrm{O}$ atomic masses, respectively. $E_{\mathrm{H}}$ is the total energy of the recoiling proton. At the first iteration, $E_{\mathrm{H}, 0}=E_{\mathrm{Si}}$. With the calculated $E_{\text {reac, } 0}$, and assuming the known initial beam energy $E_{\text {beam }}$, the vertex position was calculated using energy-loss tables [31]. From the measured vertex position, it is possible to determine the energy lost by the light particle in the gas, $d E_{0}$, before it arrives at the silicon detector. $E_{\text {reac, } 1}$ is calculated using $E_{\mathrm{H}, 1}=E_{\mathrm{Si}}+d E_{0}$ and $\theta_{\mathrm{lab}}$, leading to another vertex position. The recoil energy is corrected again $: E_{\mathrm{H}, i+1}=E_{\mathrm{Si}}+d E_{i}$. This procedure is repeated until $\left|d E_{i}-d E_{i-1}\right|<$ $1 \mathrm{keV}$. The entire procedure takes between 2 and 4 iterations. The same procedure was performed for the (p, $\alpha$ ) channel with the corresponding kinematic formula. The excitation functions at $\theta_{\mathrm{cm}}=(160 \pm 5)^{\circ}$ for both (p,p) and (p, $\alpha)$ channels are presented in Fig. 7. The absolute normalization of the data used the MWPC counter after correcting the data by the detection efficiency of the ACTAR TPC. The latter was determined using a Monte Carlo simulation that includes the geometry of the pad plane and the position of the silicon detectors. At $\theta_{\mathrm{cm}}=160^{\circ}$, the efficiency was found to vary between $90 \%$ and $100 \%$ depending on the center-of-mass energy.

\section{Results and discussion}

In order to estimate the resolution of ACTAR TPC on the center-ofmass energy reconstruction, the data were fit with a theoretical model convoluted with a Gaussian function to represent the experimental energy resolution. In the case of the (p,p) channel, the theoretical model is an R-matrix calculation performed with the AZURE2 code [35]. All resonance energies and widths that are available on ENSDF [36] were considered in the calculation. For the case of the (p, $\alpha$ ) channel, the lack of data on ENSDF led us to use a different approach. The data obtained in a previous study [34] were used as the theoretical model. 

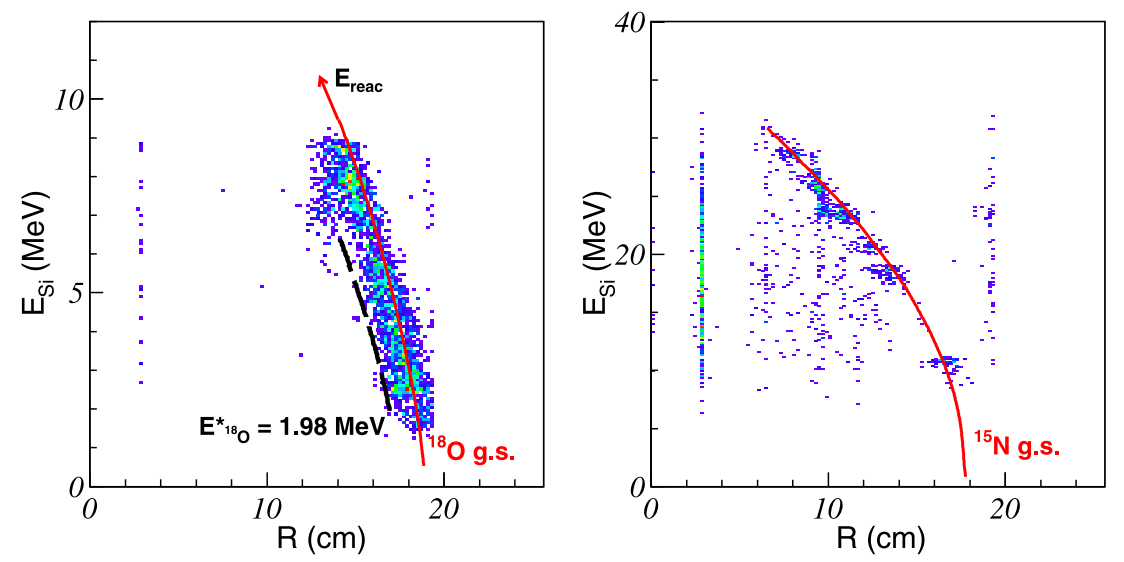

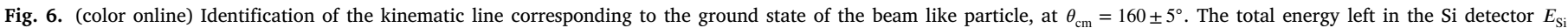
is plotted as a function of the total path $R$ (Fig. 2).
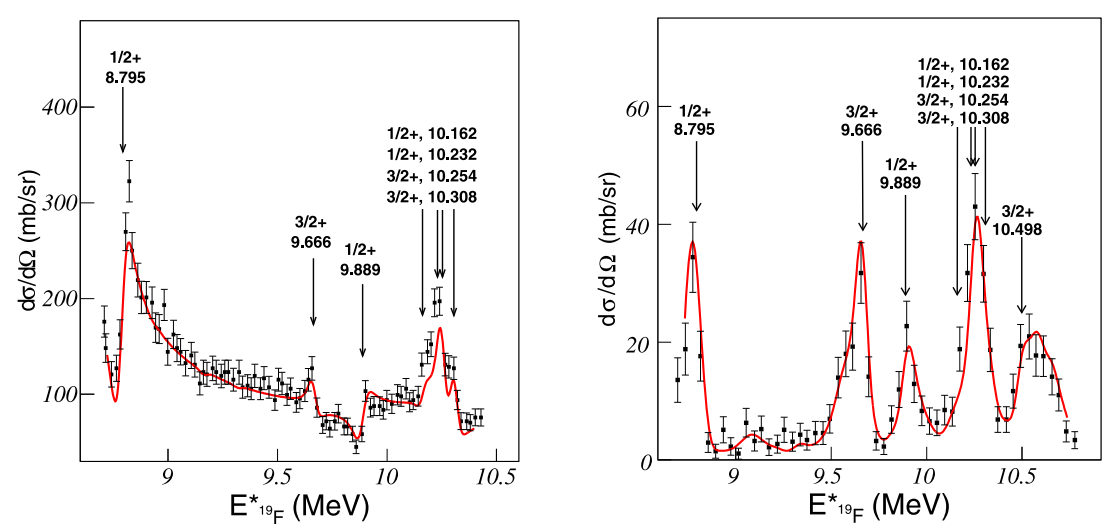

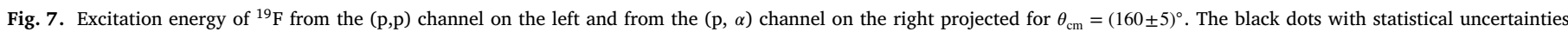

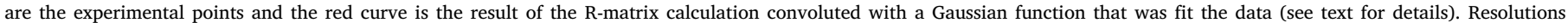
were found to be 38(4) keV FWHM and 54(9) keV FWHM, respectively.

The uncertainty on the center-of-mass energy in Ref. [34] are much smaller than in our experiment, hence the model was considered as having perfect resolution. The result of the fits are presented in Fig. 7. The resonances available on ENSDF or Ref. [34] are indicated by the arrows on the plot. Resolution on the center-of-mass energy for the $(\mathrm{p}, \mathrm{p})$ and $(\mathrm{p}, \alpha)$ channels were found to be 38(4) keV FWHM and 54(9) keV FWHM, respectively.

In order to better understand where this resolution was coming from, the uncertainties on the different experimental parameters were propagated to the center-of-mass energy determination by a MonteCarlo calculation. From Section 3.4, the parameters used to reconstruct the excitation function are:

- the beam energy at the entrance of ACTAR TPC $E_{\text {beam; }}$;

- the energy deposited by the light recoil in the silicon detectors $E_{\mathrm{Si}}$

- the light recoil diffusion angle $\theta_{\text {lab }}$.

The resolution on $E_{\text {beam }}$ primarily comes from the energy straggling produced by the $63 \mu \mathrm{m}$ thick aluminum degrader and the $6 \mu \mathrm{m}$ Mylar entrance window. This was estimated using unreacted beam events triggered by the MWPC as explained previously. The distribution of the beam reconstructed stopping point position had a lateral spread of $\sigma=6 \mathrm{~mm}$ that was translated using SRIM tables into an energy spread of $1.5 \mathrm{MeV}$ FWHM. The resolution on $E_{\mathrm{Si}}$ was determined using a ${ }^{239} \mathrm{Pu}+{ }^{241} \mathrm{Am}+{ }^{244} \mathrm{Cm}$ mixed alpha-particle source. It was assumed to vary linearly with the energy. The resolution on $E_{\mathrm{Si}}$ was found to be $60 \mathrm{keV}$ FWHM at $5.5 \mathrm{MeV}$. The resolution on $\theta_{\text {lab }}$ was measured using the shape reconstruction of the central $\mathrm{Si}$ detectors. This was achieved by tracking particles that hit the central silicon detector, and extrapolating the track to the plane of the silicon detector. The $\theta_{\text {lab }}$ resolution had a maximum value of $2^{\circ}$ FWHM. The measured resolution was estimated to be mostly due to the straggling of the recoils in the gas. For comparison, protons recorded with an energy of $3 \mathrm{MeV}$ in the Si detectors traveled at least through $175 \mathrm{~mm}$ of gas based on the reaction kinematics. When calculated with LISE++, the associated angular straggling is $1.8^{\circ} \mathrm{FWHM}$ for the proton. This is in reasonable agreement with the measured angular resolution. From the LISE++ calculations, the recoiling $\alpha$ should have a better angular resolution of $0.84^{\circ} \mathrm{FWHM}$ for a $25 \mathrm{MeV}$ recorded $\alpha$ traveling $284 \mathrm{~mm}$ of gas. The statistics on $\alpha$ particles was too small to measure the angular resolution in the same way. Since this factor has the greatest influence on the center-of-mass resolution, it was taken as $2^{\circ}$ for the (p, $\alpha$ ) channel as well so as to not underestimate its effect. A summary of the parameter resolutions and the propagation on the final simulated resolutions are given in Table 2 . The propagated uncertainties are consistent with the experimental result.

\section{Conclusion}

We have characterized the performance of a novel active target and detection system that does not rely on a magnetic spectrometer. The next generation active target ACTAR TPC was commissioned at the GANIL facility with a $3.2 \mathrm{MeV} /$ nucleon ${ }^{18} \mathrm{O}$ beam with an average beam intensity of $1.5 \times 10^{4} \mathrm{pps}$ on isobutane gas. The challenge of the energy loss discrepancy by a factor 100 between the stopping heavy-ions and the light recoils was met with the polarization of the pads below the 
Table 2

Propagation of the parameter resolution to the excitation function. All values are given in FWHM. $\theta_{\text {lab }}$ has the largest contribution to the resolution. Details on the parameter resolutions are given in the text.

\begin{tabular}{llll}
\hline Parameter & Resolution & \multicolumn{2}{l}{ Propagation to $E_{\mathrm{cm}}(\mathrm{keV})$} \\
\cline { 3 - 4 } & & $(\mathrm{p}, \mathrm{p})$ channel & $(\mathrm{p}, \alpha)$ channel \\
\hline$E_{\text {beam }}$ & $1.5 \mathrm{MeV}$ & 8.5 & 17.4 \\
$E_{\mathrm{Si}}$ & $60 \mathrm{keV}$ & 16.5 & 21.1 \\
$\theta_{\text {lab }}$ & $2^{\circ}$ & 22.3 & 34.8 \\
\hline Total simulation & & 29 & 45 \\
\hline Experimental & & $38(4)$ & $54(9)$ \\
\hline
\end{tabular}

beam. It was successful in producing two gain regions with an incident beam intensity of $20 \mathrm{kHz}$ without degrading the charge measurement. The ${ }^{1} \mathrm{H}\left({ }^{18} \mathrm{O},{ }^{18} \mathrm{O}\right){ }^{1} \mathrm{H}$ and ${ }^{1} \mathrm{H}\left({ }^{18} \mathrm{O},{ }^{15} \mathrm{~N}\right){ }^{4} \mathrm{He}$ channels were open and could be distinguished with the scattered heavy-ion identified as being in its ground state. The excitation functions in both channels were reconstructed and fit to obtain the center-of-mass energy resolutions. Resolutions of 38(4) keV FWHM for the (p,p) channel and 54(9) keV FWHM for the (p, $\alpha$ ) channel were achieved. According to simulations, the resolution is dominated by the angular resolution, which itself is dominated by the straggling of the ions in the gas. The latter could be decreased by replacing the isobutane gas target by pure hydrogen. The present resolution is comparable to the $45 \mathrm{keV}$ center-of-mass energy resolution achieved with the Active-Target Time Projection Chamber (AT-TPC) from Michigan State University that was measured for the ${ }^{1} \mathrm{H}\left({ }^{46} \mathrm{Ar},{ }^{46} \mathrm{Ar}\right){ }^{1} \mathrm{H}$ reaction $[12,37]$. This resolution was obtained with a factor 10 less beam intensity for center-of-mass angles between $30^{\circ}$ and $65^{\circ}$, and with a direct measurement of the reaction point in the active target. Using the reaction kinematics, as performed in the present work, the AT-TPC has an expected resolution of $84 \mathrm{keV}$ derived from simulations of the ${ }^{1} \mathrm{H}\left({ }^{46} \mathrm{Ar},{ }^{46} \mathrm{Ar}\right){ }^{1} \mathrm{H}$ [12]. Unfortunately, no experimental measurement was made with the AT-TPC to confirm this result due to challenges associated with the experimental data obtained in Ref. [12].

The new technological developments for active targets have opened up new possibilities to perform challenging reactions for nuclear structure and astrophysical studies. The results of the present study show that ACTAR TPC holds considerable promise in this respect.

\section{Acknowledgments}

The research leading to these results have received funding from the European Research Council under the European Union's Seventh
Framework Program (FP7/2007-2013)/ERC grant agreements $\mathrm{n}^{\circ}$ 335593 and 617156, and from the Research Foundation Flanders (FWO, Belgium) contract $n^{\circ}$ G0C $2913 \mathrm{~N}$.

\section{References}

[1] S. Beceiro-Novo, et al., Prog. Part. Nucl. Phys. 84 (2015) 124

[2] G.D. Alkhazov, et al., Phys. Rev. Lett. 78 (1997) 2313.

[3] C.-E. Demonchy, et al., Nucl. Instrum. Methods A 583 (2007) 341.

[4] M. Caamaño, et al., Phys. Rev. Lett. 99 (2007) 062502.

[5] I. Tanihata, et al., Phys. Rev. Lett. 100 (2008) 192502.

[6] T. Roger, et al., Phys. Rev. C 79 (2009) 031603(R).

[7] C. Monrozeau, et al., Phys. Rev. Lett. 100 (2008) 042501.

[8] M. Vandebrouck, et al., Phys. Rev. Lett. 113 (2014) 032504.

[9] B. Blank, et al., Nucl. Instrum. Methods A 613 (2010) 65.

[10] P. Ascher, et al., Phys. Rev. Lett. 107 (2011) 102502.

[11] E.C. Pollacco, et al., Nucl. Instrum. Methods A 887 (2018) 81-93.

[12] J. Bradt, et al., Nucl. Instrum. Methods A 875 (2017) 65-79.

[13] S. Ota, et al., J. Radioanal. Nucl. Chem. 305 (2015) 907-911.

[14] T. Furuno, et al., Nucl. Instrum. Methods A 908 (2018) 215-224.

[15] J. Hooker, et al., arXiv:1903.01402.

[16] T. Roger, et al., Nucl. Instrum. Methods A 895 (2018) 126-134.

[17] J. Giovinazzo, et al., Nucl. Instrum. Methods A 892 (2018) 114.

[18] Y. Giomataris, et al., Nucl. Instrum. Methods A 376 (1996) 29.

[19] I. Giomataris, et al., Nucl. Instrum. Methods A 560 (2006) 405.

[20] J. Pancin, et al., Nucl. Instrum. Methods A 735 (2014) 532.

[21] G. Wittwer, Clock event number transmitter receiver universal module, user's manual, GANIL July 2004.

[22] D. Suzuki, et al., Nucl. Instrum. Methods A 660 (2011) 64-68.

[23] J. Giovinazzo, et al., Nucl. Instrum. Methods A 840 (2016) 15-27.

[24] G. Jhang, Workshop on active targets and time projection chambers for high-intensity and heavy-ion beams in nuclear Physics, 2018.

[25] magboltz.web.cern.ch/magboltz/.

[26] T. Roger, et al., Nucl. Instrum. Methods A 638 (2011) 134-142.

[27] O.B. Tarasov, D. Bazin, Nuclear Phys. A 746 (2004) 411c-414c.

[28] J.F. Ziegler, J.P. Biersack, U. Littmark, The Stopping and Range of Ions in Solids, Pergamon, New York, 1985.

[29] K. Artemov, et al., Sov. J. Nucl. Phys. 52 (1990) 408.

[30] V.Z. Goldberg, A.E. Pakhomov, Phys. At. Nucl. 56 (1993) 1167.

[31] J. Ziegler, http://www.srim.org/.

[32] C.-E. Demonchy, (Ph.D. thesis), Université de Caen, 2002, https://tel. archives-ouvertes.fr/tel-00004117.

[33] T. Roger, (Ph.D. thesis), Université de Caen, 2009, https://tel.archives-ouvertes. $\mathrm{fr} / \mathrm{tel}-00424010$.

[34] R.R. Carlson, et al., Phys. Rev. 122 (1961) 607-616.

[35] R. Azuma, et al., Phys. Rev. C 81 (2010) 045805.

[36] ENSDF, http://www.nndc.bnl.gov/ensdf/.

[37] J. Bradt, et al., Phys. Lett. B 778 (2018) 155. 